\title{
Errors in LogMAR visual acuity transcription and calculation-an ongoing problem
}

\author{
Joan Hughes $^{1} \cdot$ Sophie West ${ }^{1}$
}

Received: 11 October 2019 / Accepted: 25 October 2019 / Published online: 21 November 2019

(c) The Author(s), under exclusive licence to The Royal College of Ophthalmologists 2019

We recently conducted a UK multicentre randomised controlled trial (ROSA) using Early Treatment of Diabetic Retinopathy Study best corrected Visual Acuity (VA) measurement, with LogMAR score as an outcome measure [1]. Retrospective review of source documentation identified multiple issues with calculation and recording of LogMAR scores. This finding is pertinent to Ophthalmology and Optometry teams using VA outcomes.

A total of 936 VA tests were performed for the ROSA trial across 23 UK Eye Hospitals, 2012-2017. The retrospective data review identified a mean LogMAR score recording error rate of $25 \%$.

Multiple points of error during the VA test process were identified:

- 19 tests not completed to the instructions in the Trial Specific Procedure. Of these, seven were revised following clarification of procedure. The remaining 12 (from one site) were excluded from the final dataset, due to discrepant letter and LogMAR score with no possibility of ascertaining which was correct.

- Recording letter score: Of the 22 sites included in the dataset, only 5 (23\%) showed total accuracy in recording letter score on the Case Report Form. The error rate across the remaining 17 sites ranged from 5 to $57 \%$ with transcription and addition errors. The mean letter score error rate across all 22 sites was $12 \%$.

- Recording LogMAR score: Only one site (5\%) achieved total accuracy of recording the calculated LogMAR score on the Case Report Form, with the error rate across the remaining 21 sites ranging from 6 to $79 \%$ with transcription and calculation errors. The mean LogMAR error rate across 22 sites was $25 \%$.

- No site was totally accurate across both of these variables. Independent trial data scoring was therefore undertaken.

A trial specific procedure for VA testing was provided for each site, plus a Visual Acuity source document for

Fig. 1 The LogMAR calculator

\begin{tabular}{l} 
The Newcastle upon Tyne Hospitals \\
LogMAR Calculator \\
for use with EDTRS chart \\
Type Visual Acuity Score in black box \\
(i.e. the total number of letters read at 4 metres plus 30 OR the total number of letters read at 1 metre) \\
Press Return \\
The LogMAR Score for the number of letters entered will show in the blue box \\
\hline \multicolumn{2}{|c|}{85 LogMAR Score 0.00} \\
\hline Number of Letters
\end{tabular}

Sophie West

sophie.west@nuth.nhs.uk
1 Newcastle Regional Sleep Service, Freeman Hospital, Newcastle upon Tyne, UK 
each eye to record the test. This data was transcribed locally to a Case Report Form, and sent to the trial management team. Both the VA document and trial specific procedure contained instructions for recording letters identified during VA testing and the trial specific procedure contained instructions for LogMAR score calculation. The trial specific procedure for LogMAR measurement was taken from a previous national ophthalmology research study [2].

Whilst these issues were identified in the ROSA trial, we cannot assume that they are specific to this trial, plus they have been discussed previously [3]. LogMAR score may not be routinely calculated or recorded, so it could be unfamiliar. Investigations by the authors regarding the methods of LogMAR score calculation, including discussion with trial sites and literature review failed to produce a definitive standardised approach. Use of the VA letter score as an outcome measure without LogMAR calculation has been used in some studies, perhaps because of these calculation issues. If trials intend to use LogMAR score as the outcome measure, we would advocate simpler, standardised procedures which some centres already use, such as the LogMAR Calculator (Fig. 1) and LogMAR score identification chart (Fig. 2). These facilitate accurate calculation. Further audit is required to ensure VA measurement and recording is accurate for best clinical and research decisions.
The authors report no conflicts of interest and have no proprietary interest in the materials mentioned in this article. The ROSA trial was funded by a research grant from the ResMed Foundation and ResMed UK, neither of whom were involved in the analysis or the results of the study. We are grateful to Mr Stuart Osborne, Consultant Ophthalmologist, Sunderland Eye Infirmary, for his comments.

\section{Compliance with ethical standards}

Conflict of interest The authors declare that they have no conflict of interest.

Publisher's note Springer Nature remains neutral with regard to jurisdictional claims in published maps and institutional affiliations.

\section{References}

1. West SD, Prudon B, Hughes J, Gupta R, Mohammed SB, Gerry S, et al. Continuous positive airway pressure effect on visual acuity in patients with type 2 diabetes and obstructive sleep apnoea: a multicentre randomised controlled trial. Eur Respir J. 2018;52: 1801177.

2. Jackson TL, Desai R, Simpson A, Neffendorf JE, Petrarca R, Smith $\mathrm{K}$, et al. Epimacular brachytherapy for previously treated neovascular age-related macular degeneration (MERLOT): a phase 3 randomized controlled trial. Ophthalmology 2016;123:1287-96.

3. Lovie-Kitchin JE. Is it time to confine Snellen charts to the annals of history? Ophthalmic Physiol Opt. 2015;35:631-6. 